\title{
COMPETENCIA EN INVESTIGACIÓN EN ENFERMERÍA
}

\section{RESEARCH COMPETENCE IN NURSING}

\author{
Alda Orellana Y.* \\ Olivia SanhueZa A.**
}

\begin{abstract}
RESUMEN
Enfermería como profesión y disciplina social se ve inmersa en el acelerado desarrollo de la humanidad en todos sus ámbitos, debiendo ajustarse para responder con calidad e integralidad a las nuevas necesidades de cuidado de los usuarios y de la sociedad, y adaptarse al mismo tiempo a un mundo laboral progresivamente más competitivo. La utilización de la investigación permite obtener información y generar conocimientos propios, necesarios para tomar decisiones y resolver problemas de la práctica; siendo el medio para responder a ésta y al mismo tiempo hacer visible el carácter humanista y autónomo de Enfermería. El propósito de este trabajo es examinar la competencia en investigación en enfermería aplicando los cinco elementos de una competencia desde la mirada de recursos humanos en el contexto laboral de enfermería. Se concluye que para el desarrollo de la competencia en investigación en enfermería profesional el querer hacer y el poder hacer resultan determinantes para llevar a cabo los estudios de los fenómenos de la práctica, sumados al saber, saber hacer y saber estar. Siendo responsabilidad de la institución de salud proveer los medios y la infraestructura necesarios para concretar el poder hacer dentro de la competencia en investigación.
\end{abstract}

Palabras clave: Competencia profesional, enfermería, investigación.

\begin{abstract}
Nursing as a social profession and discipline finds itself immersed in the accelerated development of mankind in every aspect, needing to adjust in order to give an integrated and quality response to the new healthcare needs of users and society, as well as adapting to an increasingly competitive labor market. The use of research allows obtaining information and generating own knowledge needed to make decisions and solve problems in the practice, being the means to respond to it and, at the same time, make the humanist and autonomous nature of nursing visible. The purpose of this paper is to examine the research competence in nursing, applying the five elements of a competence from the human resources perspective in nursing work environment. It is concluded that, for the development of competence in professional Nursing research, wanting to do and being able to, turn out to be determinants to carry out studies of the phenomena of the practice, added to the knowledge, know how to do and know how to be. It is the responsibility of the institution of health to provide the resources and the necessary infrastructure to specify the power to do in research competence.
\end{abstract}

Key words: Professional competence, nursing, research.

Fecha recepción: 12.04.10 Fecha aceptación: 19.07.10

\footnotetext{
* Doctora (C) en Enfermería. Departamento de Enfermería, Facultad de Medicina. Universidad de Concepción, Concepción, Chile.Email: aorellan@udec.cl.

** Doctora en Enfermería. Departamento de Enfermería, Facultad de Medicina. Universidad de Concepción, Concepción, Chile. Email: osanhue@udec.cl.
} 


\section{INTRODUCCIÓN}

El continuo avance de los conocimientos, el acelerado desarrollo científico y tecnológico, la globalización, las exigencias institucionales e interculturales, la comunicación por redes sociales en Internet, entre otras variables, obliga a los integrantes de la sociedad y a los profesionales a actualizarse permanentemente, y más aún, a quienes tienen la responsabilidad de formar a las nuevas generaciones de profesionales de la salud; recursos humanos que deben poseer los conocimientos y las competencias necesarias para comprender las reales necesidades de los usuarios, y dar cuenta de las exigencias que se les imponen en el mundo laboral.

El Comité Consultivo para la Formación de Enfermeros/as de la Unión Europea, destacó en 1998 que "...las competencias adquiridas al término de la formación varían ya que el ejercicio de la profesión exige que el/ la enfermero/a ingrese en un proceso de desarrollo permanente de puesta al día personal y que tenga una capacidad de evolución a fin de actualizar sus conocimientos, para poder adoptar decisiones, e incluso, introducir cambios en el ámbito profesional" $(1,2)$. Esta aseveración indudablemente remite a la competencia genérica "capacidad de investigación", competencia exigida a todo profesional $(3,4)$ y que el Consejo Internacional de Enfermeras (5) en el 2011 expresa como: "Utilizar la reflexión o la capacidad de pensar de manera crítica y realista en la práctica y ver los problemas desde un punto de vista diferente".

Actualmente, en los distintos escenarios y áreas geográficas de América se ha iniciado un movimiento que impulsa a asumir nuevos liderazgos en la disciplina de enfermería, reconociéndose que su realidad laboral es compleja y requirente de múltiples decisiones independientes, y que en Chile se ha instituido como gestión del cuidado. Esta función fue definida por la comisión de le- gislación del Colegio de Enfermeras de Chile como "la aplicación de un juicio profesional en la planificación, organización, motivación y control de la provisión de cuidados oportunos, seguros, integrales, que aseguren la continuidad de la atención y se sustenten en las políticas y lineamientos estratégicos de la institución" (6) y cuyas condiciones para su implementación y desarrollo completo han resultado más complejas de lo esperado. Algunas de las razones que podrían justificar esta complejidad es que aún persistirían dos ideas de la praxis de enfermería que se ha traducido, en algunos casos, en una equívoca delimitación del ámbito profesional de la enfermera (función), así como también en un cuestionamiento de la organización de sus servicios (estructura) (6). Por un lado, se considera que la contratación de enfermeras/ os clínicas/os por las instituciones de salud es de orden instrumental. Y por otro, que enfermería actúa bajo dependencia médica o como acota Lora-López "un instrumento del médico" (7), desconociéndose la normativa vigente del Código Sanitario (6), Libro V: De la Medicina y profesionales afines, en que se establece en forma exclusiva a las enfermeras la gestión del cuidado, función que no ha sido asignada legalmente a ningún otro profesional de la salud, comportándose entonces, como un acto propio. Esto indiscutiblemente se traduce en efectos positivos para las instituciones, como: la garantía de sus derechos y la eficiencia y eficacia en la asignación de los recursos; cuantificación de los beneficios sociales y económicos; la relación costo-efectividad y distribución de los servicios de enfermería a nivel del usuario y a nivel social (6); menor cantidad de días de hospitalización; disminución de enfermedades transmisibles; disminución en la espera para la atención; aumento de la satisfacción usuaria, entre otras.

Las modificaciones realizadas al Código Sanitario chileno (8) y la promulgación de la Ley 19.937 de Autoridad Sanitaria (9) depositaron en la enfermera/o la responsabilidad 
de la gestión del cuidado. Este reconocimiento significa, por una parte, que el cuidado es la aportación específica de la enfermera a la atención de la salud y, por otra, que un ámbito determinado de la atención en salud ha sido reservado para las enfermeras (6). Esta legislación impone documentar, justificar, sustentar científicamente y socializar los diferentes ámbitos de la práctica del cuidado de enfermería, siendo la forma, la competencia investigativa efectiva. Coincidiendo con Comet-Cortés et al., que señalan que los servicios de salud deberían apoyarse en evidencias sólidas, obtenidas desde la investigación (10).

El propósito del siguiente artículo es examinar la competencia en investigación en enfermería aplicando los cinco elementos de una competencia desde la mirada de recursos humanos en el contexto laboral de enfermería.

\section{INVESTIGACIÓN EN ENFERMERÍA}

La investigación es una función ineludible del profesional de enfermería, siendo poco valorada por las instituciones de salud, y poco exigida en el actuar de enfermería y más aún, colocada en último plano por el mismo profesional de enfermería. Las principales razones empíricas pueden ser: una baja conciencia de la importancia de la investigación, la creencia de que es un área compleja, la sobrecarga de trabajo, así como también la falta de curiosidad, perpetuando el siempre se ha hecho así, frase que coincide con autoras españolas $(11,12)$.

Afortunadamente, se está comenzando a adquirir conciencia por el conglomerado respecto de la necesidad de realizar su práctica en base a la investigación, entendida ésta como la creación de nuevo conocimiento cuyos resultados se trasladen a la sociedad, contribuyendo a mejorar su calidad de vida (13), probando a la vez teorías; experimen- tando nuevos cuidados, técnicas y procedimientos basados en costo-beneficio; exteriorizando las funciones independientes hacia los usuarios e instituciones, entre otros, lo que permitirá avanzar hacia el desarrollo de la disciplina e independencia de la profesión, resultado que identifican también Hernández et al. "Una fuerte relación entre la investigación y la práctica se traduce en mejorar el cuidado brindado a los pacientes, en el fortalecimiento del desarrollo de la profesión y en la percepción pública de enfermería" (14).

La investigación en enfermería tiene como principal objetivo "mejorar los resultados de los cuidados haciendo avanzar los conocimientos y la práctica de enfermería, e informar la política de salud" (5). El progresar en este conocimiento, es responsabilidad según Vielva del colectivo enfermería, de acuerdo a las capacidades individuales y circunstancias (15). "La educación superior y la investigación forman, hoy en día, parte fundamental del desarrollo cultural, social, económico y ecológicamente sustentable de las naciones" (16), constituyendo la investigación una demanda para el desarrollo profesional, frente a las exigencias nacionales e internacionales de posicionamiento de la profesión (3). Posicionamiento que como un círculo virtuoso se enlaza con el conocimiento, como forma de poder. Poder, reflejado a través del capital intelectual, que se observa siempre y cuando estos conocimientos sean explícitos y compartidos para que se multipliquen y no conocimientos guardados entre unos pocos (17).

Desde el espacio académico, una de las responsabilidades es producir investigación, formar investigadores en enfermería e incentivar a los estudiantes por medio de la enseñanza de una metodología de investigación amena, creativa, práctica, alcanzable y con las exigencias claras y ascendentes para cada nivel de educación. Como se concluyó en la VII Conferencia Iberoamericana de Educación en Enfermería, "el docente universitario debe promover la investigación con un compromiso real, desde la experiencia y conoci- 
miento en este oficio... estar comprometido en la promoción de la investigación... ser investigador para poder enseñar el arte u oficio de la investigación... ser maestro, el que guía, orienta, motiva, encanta, apoya desde su conocimiento y experiencia investigativa" (18). Por lo tanto, una de las innovaciones en esta área correspondería a la forma de impartir este conocimiento, mejorando las capacidades comunicativas y de interacción social, dando protagonismo a los estudiantes, haciéndose éstos responsables de su aprendizaje (19). Según Cabrero y Richard la incorporación en el currículo de la asignatura Metodología de Investigación en Enfermería en los tres niveles de enseñanza universitaria llevará a los españoles a la misma situación de otros países, pasando a ser productores de conocimientos en enfermería y no meros consumidores (20). En el estudio de Harrison, donde el $82 \%$ de las respuestas correspondió a profesoras de investigación o guías de tesis, principalmente de Chile y México, se consideró esencial para el nivel de licenciatura el conocimiento de las etapas del proceso investigativo y la realización de búsqueda bibliográfica, y la inclusión del aspecto ético en todos los niveles de formación; así como también se encontraron discrepancias en relación a tendencias de la investigación, organismos que financian las investigaciones, métodos de análisis de datos, uso de programas estadísticos computacionales, aplicación y enseñanza de la investigación, preparación de propuestas y publicaciones entre los diferentes niveles de formación (21).

\section{COMPETENCIA EN INVESTIGACIÓN}

Por investigación se utilizará la definición de Ducharme que señala: "La investigación en enfermería explora, describe, explica y predice fenómenos que son de interés para la disciplina. Permite validar y refinar los conocimientos existentes y generar nuevos que son directa o indirectamente útiles para la práctica de la enfermería” (22). Entonces investigación en enfermería sería aquella que explicita la investigación realizada por enfermeras/os y para ser utilizada en la práctica de enfermería, aunque también incluiría, a la investigación realizada por enfermeras/os en grupos multidisciplinarios donde se desea encontrar la solución o respuesta a un problema específico desde el punto de vista de cada disciplina y excluyendo aquellas investigaciones en las cuales la enfermera/o participa como colaborador en estudios que buscan resolver problemas de otras disciplinas y en las cuales generalmente su aporte es anónimo o poco reconocido cuando son socializados los resultados en el ámbito científico.

Considerando que las competencias en general surgieron en el mundo laboral/empresarial para evaluar el cumplimiento de los trabajadores asociadas a un mejor desempeño, y a una mayor calidad y productividad laboral, se utilizará la definición de competencia laboral utilizada en la Ley Chilena No 20.267 que crea el Sistema Nacional de Certificación de Competencias Laborales y perfecciona el estatuto de Capacitación y Empleo, como aquellas "aptitudes, conocimientos y destrezas necesarias para cumplir exitosamente las actividades que componen una función laboral, según estándares definidos por el sector productivo"(23). Trasladándolo al terreno práctico de la enfermería, competencia sería la aptitud, conocimiento y destreza necesarios para cumplir exitosamente las actividades que componen la función de enfermería, traducido en una mayor claridad, calidad e integralidad del cuidado de enfermería.

Con todo, competencia en investigación en enfermería sería la capacidad de la enfermera/o asistencial de demostrar aptitudes, conocimientos y destrezas necesarias para generar, validar y clarificar los conocimientos que permitan encontrar y dar solución a los problemas de la práctica de enfermería, mejorar la calidad del cuidado y al 
mismo tiempo la calidad de vida de las personas involucradas.

Según autores $(24,25)$, para que una persona pueda desarrollar los comportamientos incluidos en la competencia, es preciso que ésta tenga los siguientes elementos:

- Saber: conjunto de conocimientos que permiten realizar una cierta tarea.

- Saber hacer: aplicar los conocimientos en la solución de problemas y contar con habilidades y destrezas.

- Saber estar: ajustar los comportamientos a las normas y reglas de la organización y del grupo de trabajo. Son las actitudes e intereses.

- Querer hacer: contar con la motivación para llevar a cabo los comportamientos incluidos en determinada competencia.

- Poder hacer: se refiere a las características de la organización que permitan a la persona llevar a cabo la competencia solicitada.

Aplicando estos componentes a la competencia investigativa en enfermería se evidencia lo siguiente:

a. Saber: serían los conocimientos necesarios que permiten a la enfermera/o realizar investigación.

b. Saber hacer: la enfermera/o debe ser capaz de aplicar sus conocimientos en investigación, tener habilidad y destreza para dar solución a los problemas y dudas derivadas de la práctica de enfermería.

c. Saber estar: la investigación debe ajustarse a las normas éticas, intereses de la institución de salud y mantener una actitud científica.

d. Querer hacer: la enfermera/o debe querer realizar investigación, siendo el principal impulsor de la investigación la motivación personal para llevarla a cabo.

e. Poder hacer: la enfermera motivada e interesada en realizar investigación debe contar en su institución con los recursos y medios (económicos, computacionales, tiempo, etc.) que sean necesarios para llevar a cabo la investigación.

Se entiende que para desarrollar investigación en la práctica es básico que cada uno de estos elementos se materialicen en el profesional de enfermería, en otras palabras, "ser competente en investigación va a implicar el dominio de la totalidad de estos elementos y no sólo de alguna de las partes" (26).

De los anteriores, el querer hacer se muestra relevante, ya que la/el enfermera/o puede manejar con diferente grado de profundidad todos los aspectos relativos a producir investigación (metodología de investigación, bioética, lectura crítica, entre otros), según el nivel de estudios alcanzado; puede contar con el apoyo institucional; puede tener los recursos, pero falla la intencionalidad, no desea ni tiene la intención de aplicarlos en su contexto diario de trabajo. Bolaños y Sánchez al respecto opinan que "Investigar es una función genérica de la disciplina de enfermería e implica involucrar a todos los miembros" (27), asumiendo que deben incluirse todas/os las/os enfermeras/os interesados, pero si no hay motivación personal no existirá investigación.

Especificar el nivel de esta competencia investigativa, según los distintos niveles de formación de profesionales de enfermería, resulta aclaradora y necesaria para la práctica:

- El nivel de Licenciado en enfermería debiera contar con los conocimientos del proceso de investigación científica, de los principios éticos aplicados a la investigación y de la realización de búsqueda bibliográfica (21), de tal manera de poder participar en grupos de investigación como colaborador y a la vez proponer temas de investigación derivados de la práctica diaria, "formular preguntas interesantes y relevantes para la enfermería" (28). $\mathrm{El} / \mathrm{la}$ Licenciado/da en enfermería debiera 
estar preparado/a para llevar a cabo investigaciones de tipo descriptivo en un grupo de investigadores con experiencia, y poseer habilidad en búsqueda de información y lectura crítica, para reconocer la validez de las investigaciones y aplicar sus resultados (práctica de enfermería basada en la evidencia). Otros autores incorporan además para el nivel de Licenciado/a la capacidad de realizar estudios de casos (27).

Las enfermeras/os de nivel de Magíster debieran poseer los conocimientos y habilidades anteriores en mayor profundidad y complejidad, participando activamente en grupos multidisciplinarios de investigación. Integrar la investigación a la docencia y/o la clínica para hacer de la "investigación un lugar de encuentro entre profesionales de enfermería asistenciales y docentes" (15), promoviendo líneas de investigación de acuerdo a las necesidades presentes en la asistencia, aplicar los resultados de investigación en la práctica, realizar y replicar investigaciones de tipo descriptivo, explicativo y experimentales para optimizar la gestión del cuidado.

- El nivel Doctorado en enfermería debiera crear y liderar líneas de investigación en los escenarios de Enfermería en equipos multidisciplinarios; aplicar la metodología cuantitativa y/o cualitativa e integrar investigadores cualitativos en los equipos o núcleos de investigación. Este último abordaje es esencial para comprender los fenómenos de enfermería, lo que le permitirá la creación de conocimiento y por lo tanto el desarrollo de la ciencia de enfermería. La/el enfermera/o que realiza investigación en la práctica debe contar en el ámbito laboral con hemerotecas con revistas científicas actualizadas, con medios informáticos, bases de datos, y con el tiempo necesario para cumplir con dicha función.

Dentro de la competencia en investi- gación en enfermería se incluyó varios dominios como es la metodología de investigación; principios éticos; búsqueda de información y lectura crítica, sin mencionar aún la comprensión idiomática. Gran parte de la investigación publicada en enfermería es realizada por enfermeras de habla inglesa, quienes han aportado enormemente a la producción científica propia de enfermería. Por consiguiente, el manejo del inglés técnico debe formar parte de esta competencia para realizar una búsqueda y lectura exhaustiva de la información.

La competencia investigativa comprende la socialización de los resultados de las investigaciones, puesto que muchas investigaciones son metodológicamente válidas y sus resultados útiles para la práctica de enfermería, no siendo dados a conocer a la comunidad científica. La socialización incluye presentaciones en eventos científicos y publicación en revistas de corte disciplinar. Lora-López expone que la competencia en investigación tiene relación con la presentación de los resultados de manera adecuada a una audiencia (7).

Muchas investigaciones de enfermería se socializan principalmente en congresos o eventos científicos o académicos, lo que permite de cierto modo a investigadores noveles conocer las etapas y resultados de la investigación, siendo además una instancia motivadora. Sin embargo, para llegar a ser conocidos estos resultados por una masiva concurrencia de profesionales es necesario otra serie de pasos que no siempre las/os enfermeras/os están dispuestas/os a realizar, como es la elaboración del artículo científico. Dicho artículo permite a los lectores conocer la metodología utilizada, los resultados y las conclusiones de la investigación, y por tanto, los beneficios de la aplicación de los resultados. No obstante, este paso muchas veces no se llega a concretar porque no se 
considera significativo difundir los resultados fuera del contexto laboral; o también porque el desarrollo de un artículo científico presenta la dificultad de plasmar y resumir en detalle y claramente las etapas llevadas a cabo en la investigación. Escribir un artículo científico requiere dominios que deben adquirirse a lo largo del tiempo, con formación en escritura científica o bajo la guía de investigadores con experiencia. Morales et al. opinan que los estudiantes universitarios requieren formación en capacidad discursiva con la escritura de múltiples borradores (29).

\section{CONSIDERACIONES FINALES}

Es a través de la investigación que el conocimiento de enfermería puede ser depurado al punto de atender con calidad las crecientes y cambiantes necesidades de atención en salud presentadas por las personas, y acompañar con éxito los cambios en los sistemas de salud y viceversa. Enfermería se hará visible a los ojos de las propias profesionales de enfermería como de la sociedad toda, al hacer evidente la eficacia, efectividad, eficiencia, pertinencia, seguridad e integralidad de los cuidados entregados a los usuarios.

Se propone la definición de competencia en investigación que nace desde el punto de vista laboral de enfermería, donde se suma a los saberes utilizados en el mundo educacional, el querer hacer y el poder hacer, donde la motivación y el apoyo institucional respectivamente son imprescindibles para realizar investigación. Contar entonces con competencia en investigación es un requisito primordial en el momento actual para apropiarse y empoderarse del nuevo modelo de cuidado, que permita la transformación cultural de transitar hacia una práctica basada en evidencia que entrelaza la ciencia, la ética, la filosofía y la práctica. Competencia en investigación que debe correlacionarse con el grado de formación en enfermería, el que va entregando diferentes niveles de responsabilidad y donde los postgrados son el motor principal para estimular y motivar a los estudiantes, y a través de ellos a la comunidad de enfermería.

A medida que se apliquen más intervenciones basadas en investigación, habrá mayor necesidad de crear conocimiento, potenciando a Enfermería como disciplina humanista y revelando nítidamente el carácter autónomo y social que la profesión posee.

\section{REFERENCIAS}

1. Sociedad Española de Enfermería de Urgencias y Emergencias. Competencias de Enfermería en Urgencias y Emergencias. [Internet]; año desconocido [citado 3 Oct 2008]. Disponible en: http://www. enfermeriadeurgencias.com/documentos/fundamentos/competencias.pdf

2. Falcó A. La nueva formación de profesionales: sobre la competencia profesional y la competencia del estudiante de enfermería. Edu Med Salud. 2004; 7(1):42-5.

3. Universidad de Concepción. Dirección de Docencia. Boletín No 1 [Internet]; 2008. [citado 3 Oct 2008]. Disponible en: http:// docencia.udec.cl/images/documentos/ BOLETIN_N011.pdf

4. Tunning-América Latina. Reflexiones y perspectivas de la Educación Superior en América Latina. Informe final Proyecto Tuning América Latina 2004-2007 [Internet]; 2007 [citado 3 Oct 2008]. Disponible en: http://tuning.unideusto.org/ tuningal/index.php?

5. Consejo Internacional de Enfermeras. Resolver la desigualdad: aumentar el acceso y equidad [Internet]. [Ginebra]: Consejo Internacional de Enfermeras; 2011 [citado 2 Oct 2008]. Disponible en: http://www.icn.ch/es/publications/international-nurses-day/ 
6. Milos P, Bohórquez B, Larrain A. La "gestión del cuidado" en la legislación chilena: Interpretación y alcance. Cienc enferm. 2010; XVI (1): 17-29.

7. Lora-López P. Reflexiones sobre el grado y postgrado de Enfermería: la investigación en Enfermería [editorial]. Index Enferm. 2008;17(2): 85-6.

8. República de Chile. Código Sanitario. 12a edición oficial. Santiago, Chile: Editorial Jurídica de Chile; 2006. Libro quinto del ejercicio de la medicina y profesionales afines. Artículo 113; p.35.

9. Ley 19.937 de 30 enero, 2004. Autoridad Sanitaria. Modifica el D.L. No 2.763, de 1979, con la finalidad de establecer una nueva concepción de la autoridad sanitaria, distintas modalidades de gestión y fortalecer la participación ciudadana. Diario Oficial Chile 24 febrero 2004.

10. Comet-Cortés P, Escobar-Aguilar G, González-Gil T, De Ormijana-Sáenz A, Rich-Ruiz M, Vidal-Thomas C et al. Establecimiento de prioridades de investigación en enfermería en España: estudio Delphi. Enferm Clin. 2010; 20(2): 88-96.

11. Benavides M, Rodríguez M. El porqué de las cosas. Evidencias de una reflexión Enfermera [carta]. Enferm Com [Internet]. 2008 [citada 12 Dic 2010]; 4(2). Disponible en: http://www.index-f.com/comunitaria/v4n2/ec6854.php

12. De la Concepción M, Fernández J, Gómez C, López C, Rionda M. La enfermería y los cuidados de los catéteres intravasculares. Excelencia Enfermera [Internet]. 2005 [citada 12 Dic 2010]; (9). Disponible en: http://www.ee.isics.es

13. Universidad de Concepción. Plan Estratégico Institucional [Internet]; 20062010 [citado 12 Dic 2010]. Disponible en: http://www.udec.cl/ppei20062010/ index.html

14. Hernández M, Gómez B, Becerril L, Rojas A. Cuidados de la Salud: paradigma del personal de enfermeros en MéxicoLa reconstrucción del camino. Esc Anna
Nery Rev Enferm. 2009;13(2):287-96.

15. Vielva J. La docencia de pregrado, la función asistencial y la investigación enfermera [editorial]. Boletín IDEA. [Internet]. 2006 [citado 12 Dic 2010]; 11. Disponible en: http://www.madrid.org/cs/

16. Jara P, Stiepovic J. Currículo por competencias en el postgrado de enfermería. Invest Educ Enferm. 2007;25(2):122-129.

17. Pereda S, Berrocal F. La gestión del conocimiento. En: Gestión de recursos humanos por competencias. 2a. edición. Madrid: Editorial Universitaria Ramón Areces; 2006. p. 87-107.

18. Romero MN. Conclusiones de Simposio Formación de investigadores de VII Conferencia Iberoamericana de Educación en Enfermería. VII Conferencia Iberoamericana de Educación en Enfermería. [Internet]. 2003 [citado 29 Oct 2008]. Disponible en: http://www.aladefe.org/ index_files/docs/conclusiones_cidee/formacion_investigadores_enfermeros.doc

19. Irigoin M, Vargas F. La formación basada en competencias. En: Competencia laboral: manual de conceptos, métodos y aplicaciones en el sector Salud. Montevideo: Cinterfor; 2002. p. 217- 40.

20. Cabrero J, Richart M. Concepto de investigación en enfermería. En: Investigar en enfermería. Concepto y estado actual de la investigación enfermería. Alicante: Publicaciones Universidad de Alicante; 2005. p. 17- 48.

21. Harrison L, Ray A, Cianelli R, Rivera M, Urrutia M. Competencias en investigación para diferentes niveles de formación de enfermeras: una perspectiva latinoamericana. Cienc enferm. 2005;11(1):5971.

22. Ducharme F. La recherche en sciences infirmiers, des défis à relever. Can J Nurs Res. 1997; 29(4):123- 40.

23. Biblioteca del Congreso Nacional de Chile. Ley 20.267 de 25 junio, 2008. Crea el sistema nacional de certificación de competencias laborales y perfecciona el esta- 
tuto de capacitación y empleo. Ministerio del trabajo y previsión social [citado 3 Oct 2009]. Disponible en: http://www. leychile.cl/Navegar?idNorma $=272829$

24. Pereda S, Berrocal F. La gestión de recursos humanos por competencias. En: Gestión de recursos humanos por competencias. 2a. edición. Madrid: Editorial Universitaria Ramón Areces; 2006. p. 69-86.

25. Pereda S, Berrocal F. Las competencias. En: Técnicas de gestión de recursos humanos por competencias. 2a. edición. Madrid: Editorial Universitaria Ramón Areces; 2005. p. 17-35.

26. González L, López L. La sociedad del conocimiento y la formación de profesio- nales. En: Centro Universitario de Desarrollo, eds. Competencias de egresados universitarios. Santiago, Chile: Centro Universitario de Desarrollo; 2004. p. 25164.

27. Bolaños J, Sánchez T. Nivel de competencia de enfermería en investigación. Rev Enferm IMSS. 2000; 8(3):155-9.

28. Abreu A. Formación permanente en enfermería e investigación. ¿Una manera de definir nuestro rol? Tempos Vitales. Revista Internacional para el Cuidado del Paciente Crítico. 2004; 4(1):14-7.

29. Morales O, Rincón A, Tona J. Cómo enseñar a investigar en la universidad. Educere. 2005;9(29):217-25. 